\title{
Recent advances in understanding and managing Chlamydia trachomatis infections
}

\author{
Jeanne Marrazzo* and Robert Suchland
}

\author{
Address: Department of Medicine, University of Washington, Box 359932, 325 Ninth Avenue, Seattle, WA 98104, USA \\ *Corresponding author: Jeanne Marrazzo (jmm2@uw.edu) \\ Fl000Prime Reports 2014, 6:120 (doi:10.12703/P6-120) \\ All FI000Prime Reports articles are distributed under the terms of the Creative Commons Attribution-Non Commercial License \\ (http://creativecommons.org/licenses/by-nc/3.0/legalcode), which permits non-commercial use, distribution, and reproduction in any medium, \\ provided the original work is properly cited. \\ The electronic version of this article is the complete one and can be found at: http://fl000.com/prime/reports/m/6//20
}

\begin{abstract}
Worldwide, Chlamydia trachomatis infections rank among the most common sexually transmitted infections (STI), and cause notable reproductive morbidity in women. Although advances in highly accurate and non-invasive diagnostic testing have allowed for better estimation of the burden of disease - especially the asymptomatic state-we still lack a true point-of-care test, and many infections go undetected and untreated. Moreover, limited resources and effort for managing sexual partners of those in whom infection is actually identified comprise a major challenge to control. Here, we review the current state of understanding of this common infection, and efforts to control it.
\end{abstract}

\section{Introduction}

C. trachomatis is the most commonly reported bacterial infection in the US and the most common cause of urethritis in men and cervicitis in women, and certain strains can cause severe proctitis in men who have sex with men (MSM) [1]. Because most infections cause neither symptoms nor signs-especially in women, $90 \%$ of whom are asymptomatic-a large reservoir of untreated persons provides an ongoing source for efficient disease transmission. Unfortunately, the asymptomatic nature of chlamydial infections in the female reproductive tract facilitates the silent immunopathogenesis that causes scarring of the Fallopian tubes, ovaries, endometrial lining, and occasionally the adjacent perineum, promoting future ectopic pregnancy and tubal infertility. For this reason, C. trachomatis is thought to be the most costly non-viral sexually transmitted infection STI, with total lifetime direct medical costs estimated at $\$ 516.7$ million [2].

Screening programs aimed at preventing pelvic inflammatory disease (PID) in women began in the late 1980s and were endorsed by the US Preventive Screening Task Force (USPSTF) and the Centers for Disease Control and Prevention (CDC) in the mid-1990s [3]. Although the adverse sequelae of chlamydial infections in women have generally declined in countries with established screening programs, generalized prevalence has not-an observation that has prompted several intriguing hypothetical explanations. Has there been a shift in the natural history of the pathogen? Has screening promoted a shorter duration of infection and thus modified the host immune response? Have screening programs simply continued to target new populations at high risk over time? Is single-dose treatment of uncomplicated genital infections with azithromycin-widely used-adequate? These questions have generated considerable controversy, and the answers to them are critical to informing our future approach to control of this important STI.

\section{Current epidemiologic trends}

In 2012, 1,422,976 chlamydial infections were reported to the CDC in 50 states and Washington, DC-a rate of 456.7 cases per 100,000 population-remarkably, the largest number of cases of any disease ever reported to the CDC during a year's time [1]. However, these surveillance data had encouraging trends. The case count represented a small increase (only $0.7 \%$ ) from the prior year, the smallest such increase recorded since this surveillance began. Also, the rate in women did not increase from 
the prior year. However, the overall rate of infection in the US in women (643.3 cases per 100,000 women) was more than twice that among men (262.6 cases per 100,000 men), likely reflecting the fact that women are considerably more likely to be screened than are men. More men are probably being tested as urine-based assays are increasingly used: the CDC STD Surveillance Report notes that, over the years of 2008 to 2012, the chlamydia rate in men increased by $25 \%$. Because $90 \%$ of cervical infections in women cause neither signs nor symptoms and at least $60 \%$ of urethral infections in men are asymptomatic, these rates are undoubtedly an underestimate of the true burden of disease.

Selective screening of appropriate women is necessary to control this infection and its sequelae, and most experts agree that it has effected widespread declines in reproductive tract sequelae; whether it has effected declines in prevalent infection is a question of debate [4]. The USPSTF and the CDC recommend that all sexually active women age 25 years or younger be screened annually for C. trachomatis and that screening of older women be based on behavioral risk criteria [5,6]. Despite this, rates of appropriate screening in young women remain suboptimal, and interventions to enhance screening in target populations are needed [7].

Some features of chlamydia epidemiology have remained constant since surveillance was initiated in the US. Prevalence is consistently highest among young women (15 to 24 years of age), and rates peak among men at a slightly older age (20 to 24 years) [1]. In one prospective study of a cohort of 14,322 individuals between the ages of 18 and 26 years, the prevalence of chlamydial infection was $4.2 \%$ [8]. The highest rates were in African-American women $(14 \%)$ and overall were higher in women than men. Some experts believe that a decline in prevalence with age may be related to the development of partial immunity to clinical infection through periodic repeated exposures as well as to changes in behavior $[9,10]$.

In both men and women, race, ethnicity, economic disadvantage, and geographic region modify the likelihood of infection being detected. For example, in 2012, the rate in blacks was over seven times the rate in whites. State-specific prevalence was highest in Alaska, where disease is heavily focused in the Native Alaskan population. Among women, prevalence was very high among those economically disadvantaged women who were 16 to 24 years old and who entered the National Job Training Program; median state-specific prevalence was $11.0 \%$ (range $5.5 \%$ to $19.4 \%$ ). Among men entering this program, median state-specific prevalence was $7.0 \%$ (range $0.6 \%$ to $13.5 \%$ ).

\section{Global epidemiology}

Few nationally representative data-as opposed to convenience samples-are available for most countries in the world. The World Health Organization estimated that, in 2008, 105.7 million new cases of chlamydia occurred worldwide, a $4.1 \%$ increase since the last comprehensive assessment, in 2005 [11]. A population-based study in China estimated the prevalence of $C$. trachomatis infection at the surprisingly high rate of 2.1 per 100 population; unprotected sex with a commercial sex worker was a significant risk factor for infection among men from 20 to 44 years of age [12]. One interesting feature of recent C. trachomatis epidemiology is the so-called Swedish new variant, first reported in 2006 in Sweden and initially undetected by the nucleic acid amplification test (NAAT) in use at the time, thus allowing infections caused by this strain to go undetected and cause a nationwide network of related infections [13]. Subsequent modification of testing protocols has resulted in relatively lower proportion of chlamydial infections due to this organism, and it remains most common in the Nordic countries.

\section{Sexual minority populations}

C. trachomatis is likely to be diagnosed as an asymptomatic infection through routine annual screening at the rectal site or as a cause of proctitis in men who engage in receptive anal intercourse with other men in the form of lymphogranuloma venereum (LGV). Whereas uncomplicated genital chlamydial infections are caused by C. trachomatis serovars D-K, LGV is caused by serovars L1, L2, and L3. LGV strains of C. trachomatis continue to cause discrete outbreaks of ulcerative proctitis in industrialized countries, and some data suggest that this pathogen is not sought as a cause of proctitis as often as is warranted [14-16]. Nearly all cases reported in such outbreaks are among HIV-infected MSM who report unprotected receptive anal sex, and the L2 serovar is the dominant pathogen. In an outbreak of LGV described in the UK among 282 MSM, the majority (96\%) had proctitis with either severe or systemic symptoms, and there was a high level of coinfection documented with HIV (76\%), hepatitis C (19\%), and other STIs (39\%) [15].

Despite the clinical picture associated with LGV, the majority of rectal chlamydial infections, especially nonLGV, are asymptomatic [17]. Moreover, in the most recent study, $85 \%$ of chlamydial infections in this population of MSM occurred at the rectum and would have been missed if only urethral screening was performed; thus, routine screening of sexually exposed anatomic sites (urethra and rectum) should be performed in MSM.

Women who were 15 to 24 years old, who were attending family planning clinics in the US Pacific Northwest during 
1997 through 2005, and who reported same-sex behavior had higher positivity of $C$. trachomatis than women who reported exclusively heterosexual behavior [18]. Of note, there was also high chlamydia positivity among American Indian/Alaska Native women who reported sex with women, a finding consistent with racial/ethnic disparities previously noted. Possible explanations for this observation relate to differences in these two groups' use of reproductive health-care services (including chlamydia screening), biological susceptibility to lower genital tract infection, infrequent use of barrier methods to prevent STI transmission with female partners, trends toward higher risk behavior, and differential characteristics of their respective sexual networks.

\section{Natural history and the immune response}

The natural history of $C$. trachomatis varies, and efforts to define its determinants are ongoing, particularly in light of declines in incidence of its complications in women. Several prospective studies have documented high rates of repeat infection in the months after an initial chlamydial infection. In the most rigorous of these, test-of-cure was performed approximately 30 days after treatment to document antibiotic cure; participants then returned at 3 months for repeat screening, thus documenting that repeat infection actually occurred. Among 272 men followed for 4 months after a diagnosis of chlamydial infection in Baltimore, Denver, and San Francisco, repeat infection occurred in 13\% (incidence, 45.4 infections per 100 person-years) [19]. Among 897 female adolescents attending school-based health centers, 236 had one or more subsequent positive tests for a cumulative incidence of reinfection in one year of $26.3 \%$ (95\% confidence interval [CI] 23.4 to 29.2) [20]. Rescreening several months after a diagnosis of chlamydia is recommended as a population-level prevention method. This intervention, unfortunately, is underutilized [21] and should be prioritized for widespread benefit.

Some investigators have hypothesized that high rates of reinfection are due in part to antibiotic therapy being provided, on average, earlier in the course of infection than would have been the case had screening programs not been in place. This "arrested immunity hypothesis" suggests that early antibiotic treatment effectively attenuates the optimal development of protective immunity, leaving individuals as susceptible as before to reinfection with the same or a new serovar $[9,10]$. Population-based surveillance data certainly support high rates of reinfection. The most relevant animal data are from the murine model, which show that when primary chlamydial infection resolves in the absence of antibiotic therapy, the animals show evidence of immunity against subsequent challenge [22,23]. Geisler and colleagues evaluated whether spontaneous resolution of chlamydial infection in humans was associated with decreased reinfection in women returning for treatment for a positive chlamydia screening test [24]. Of the women who experienced spontaneous resolution, reinfection was less frequent relative to those women who had persistent infection detected at follow-up (19.9\% versus $4.5 \%$; $P=0.016)$. Again, these data suggest that treatment may attenuate protective immunity in some persons and conversely that natural immunity may protect against reinfection.

The main question that remains is how much reinfection is attributable to these biologic mechanisms or simply to a high likelihood of re-exposure to untreated (or inadequately treated) sex partners. There is no doubt that rates of successful partner treatment-discussed below-remain suboptimal and contribute to this problem.

\section{Screening and testing to control disease}

Because the minority of chlamydial infections present with symptoms, screening of asymptomatic persons plays a critical role in detecting the majority of infections. The premise of selective screening approaches relies on the identification of risks that are specific and sensitive for detection of asymptomatic infection. In the 1990s, a randomized controlled trial designed to determine whether selective screening for chlamydial infection reduces the risk of subsequent PID showed that women randomly assigned to undergo screening had a $56 \%$ lower rate of PID over the following year than did women receiving the usual care without screening [25]. This report helped prompt US national guidelines for risk-based chlamydial screening of young women to reduce the incidence of PID and the prevalence of postPID sequelae while also reducing sexual transmission of C. trachomatis. The CDC and the USPSTF recommend that sexually active women of not more than 25 years of age be screened for genital chlamydial infection annually $[5,6]$. Later studies that examined this issue generally support the benefits of this intervention; one study that did not, the POPI (Prevention of Pelvic Infection) Trial, had serious limitations, the most significant being low statistical power for the end-points of interest (PID incidence overall was $1.6 \%$ ) and incomplete ascertainment of the microbiologic and clinical end-points (only $17 \%$ of women defined as having PID had this documented by medical record review) [26]. Despite the recommendations for routine screening and despite widespread availability of accurate NAATs that can be performed on urine and self-collected vaginal swabs without the need for pelvic examination [27], screening coverage in many primary care settings remains low. 
Retesting is performed for the express purpose of evaluating whether a patient has been reinfected. The majority of recurrent chlamydial infections result from resumption of unprotected sex with an untreated sex partner, although some do result from sex with a new sex partner. In women, repeat infections confer an increased risk of developing complicated genital infections, such as pelvic inflammatory disease. Thus, recently infected persons should have repeat testing for $C$. trachomatis infection approximately 3 to 6 months after treatment, regardless of whether patients believe their sex partners were treated.

\section{Treatment}

Treatment of C. trachomatis infection requires special consideration, largely due to its somewhat unique life cycle. The infectious form of the organism, the extracellular elementary body, is metabolically inert and resistant to killing. Thus, antibiotics must target the sequestered intracellular and intravacuolar phases of the life cycle of this pathogen. For this reason, antibiotics with good intracellular penetration must be used. Antibiotic concentrations must be present throughout the entire 36- to 48-hour life cycle of the organism. Thus, either a prolonged course of therapy or selection of an antibiotic with a long half-life is required to ensure adequate levels of the antibiotic agent. Azithromycin, the prototype for chlamydia treatment, has excellent intracellular and tissue penetration. Owing to its half-life of 5 to 7 days, it can be administered as single-dose therapy (1 gram orally) under direct observation and can be used in pregnant women.

More recently, there has been debate about the relative efficacy of the azithromycin and doxycycline regimens. In a large randomized controlled trial of different antibiotic regimens in men with non-gonococcal urethritis, the microbial clearance rates among 115 men with C. trachomatis were $94.8 \%$ for the doxycycline arm and $77.4 \%$ for the azithromycin arm [28]. A recent meta-analysis of 23 randomized trials including over 2000 patients compared the efficacy and safety of doxycycline and azithromycin for treatment of uncomplicated genital chlamydial infections in men and women [29]. The analysis found a pooled efficacy in favor of doxycycline of $1.5 \%$, with a larger estimate for symptomatic men (7.4\%). Although an earlier study had documented very close microbial cure rates between both agents (97\% and $98 \%$ for azithromycin and doxycycline, respectively), most of the microbiological cure in these earlier studies was documented with culture or enzyme immunoassay testing rather than the more sensitive NAAT assay [30]. The implications of these findings are not clear, and the quality of the studies included varied widely. The response of expert opinion about a possible threat has ranged from acknowledgment of a need for more data to suggestions that study of longer or enhanced dosing regimens of azithromycin be considered [31,32].

These concerns notwithstanding, antibiotic resistance appears to be exceedingly rare in C. trachomatis. In fact, the Chlamydia species has been such a successful pathogen most likely due to its intricate but elegant life cycle (Figures 1 and 2). The intracellular nature of the pathogen eliminates the opportunity for rapid evolution of cell surface components that could contribute to drug resistance. Furthermore, the elementary body is relatively inert and this limits opportunities for replication and the generation of antibiotic-resistant mutations. However, spontaneous antibiotic-resistant mutations are relatively easily selected for in in vitro cell culture, and lateral gene transfer of tetracycline resistance has been demonstrated [33]. These findings point to the fact that $C$. trachomatis has the biological potential to acquire antibiotic resistance. These features may also explain the paucity of inflammatory responses and the asymptomatic nature of most chlamydial infections. That said, new antibiotic options are always welcome and eventually needed. A recent proof-ofconcept clinical trial found that the microbiologic cure rate of single-dose oral rifalazil $(25 \mathrm{mg}$ ) was not noninferior to that seen with standard azithromycin therapy; cure rates, respectively, were $84.8 \%$ versus $92.1 \%$, the difference in cure rate was $7.3 \%$, and a lower limit of the $95 \%$ CI was 22.5 ; thus, non-inferiority was not established at the prespecified margin (lower limit of CI of $15 \%$ ). However, this was a proof-of-concept study, and the number of treatment failures at a midstudy secondary end-point was not different [34]. Larger studies are required to pursue this potential therapeutic option.

\section{Role of management of sex partners}

Unfortunately, rates of successful treatment of sex partners of persons with C. trachomatis remain low, and resources to detail public health staff to assist in partner notification efforts are not available. In the absence of such efforts, patients are often asked to notify their partners of the need for medical evaluation and treatment. This approach has been deemed a public health failure. A strategy termed "expedited partner management" (EPM) is an alternative way to increase the proportion of sex partners receiving treatment and to decrease rates of reinfection in the index patient. With $E P M$, treatment (without examination) is facilitated by giving an antibiotic or a prescription to the index patient or by calling in a prescription directly for the 
Figure I. Developed Chlamydial inclusion

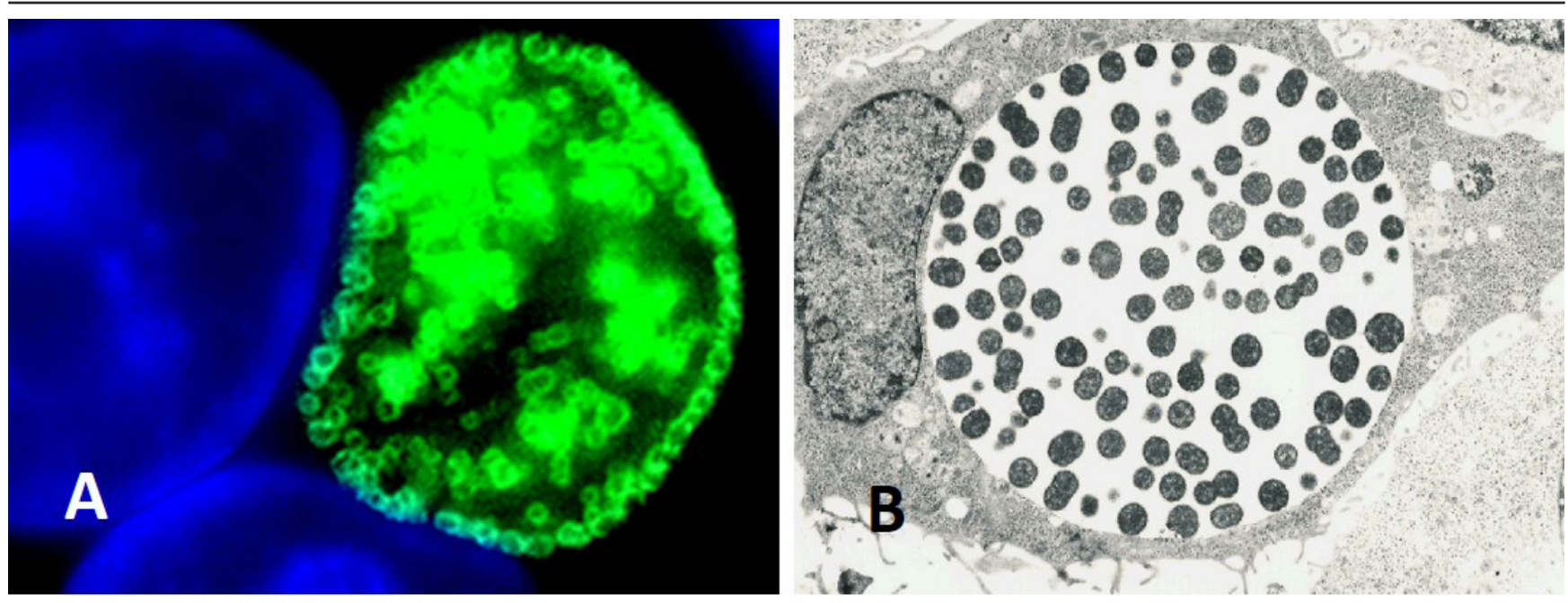

Images of developed Chlamydia trachomatis inclusions in human epithelial cells. (A) Immunofluorescent image demonstrating Chlamydia reticulate bodies (green) located near the periphery of the inclusion at 30 hours post infection. The inclusion can be seen to be pressed up next to the cell nucleus (blue). (B) Electron micrograph of a similar inclusion at 40 hours post infection with reticulate bodies next to the inclusion membrane as well as elementary and intermediate bodies within the inclusion. Copyright @ American Society for Microbiology, Infection and Immunity, 68, 2000, 360-367, and doi: I0.II28/IAI.68.I.360-367.2000

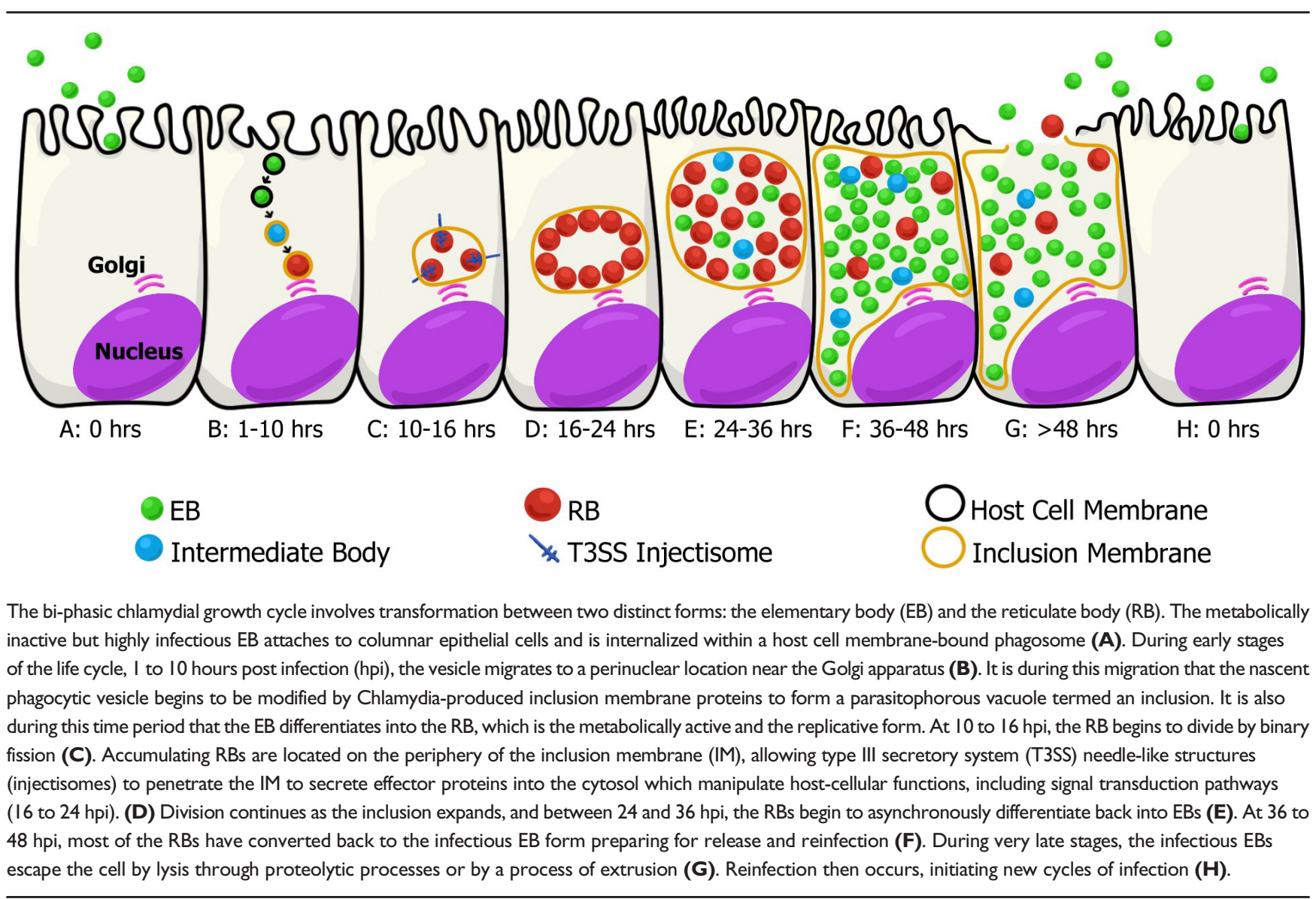


partner. Expedited partner therapy (EPT) for chlamydia can be accomplished with single-dose oral therapy (azithromycin). Patients and sexual partners should be counseled to abstain from sexual activity until the therapeutic course of antibiotics is completed or, in the case of azithromycin, for 7 days after the single-dose treatment was administered.

In one randomized controlled trial, 2751 patients $(23 \%$ male) with diagnosed gonococcal infection, chlamydial infection, or both were assigned to either an "expedited treatment group" or a standard referral group [35]. Patients in the expedited treatment group were given "partner packets" of cefixime or azithromycin (or both) to distribute to their partners, without requiring a formal medical evaluation of the partners. In the standard referral arm, patients simply notified their sex partners of the need for evaluation and treatment. Patients assigned to expedited treatment of sexual partners were significantly more likely to report that all of their partners had been treated compared with those assigned to the standard referral arm (61\% versus $49 \%)$. The trial also demonstrated a significant benefit in lowering the number of recurrent infections among those assigned to expedited treatment (relative risk of reinfection 0.75 , $95 \%$ CI 0.56 to 0.97$)$.

Clinicians should routinely employ EPT for the partners of persons with chlamydial infection, to the extent permitted by regional laws and regulations where they are practicing, when follow-up is in question. The legal status of EPT is state-specific, although surveys indicate that many clinicians frequently pursue the practice in managing their patients with STIs. The legal status of EPT options can be found at www.cdc.gov/std/ept.

\section{Conclusions}

Despite major advances in the diagnosis and management of chlamydial infections, we are still faced with several conundrums. Among the most important is how to plan for the future of effective screening programs that will guarantee that low rates of upper reproductive tract sequelae of untreated infection remain minimized. In the face of sustained positivity among the groups now being screened, can we successfully argue to funders and policymakers that screening is working? How can we communicate, and rigorously document, the impact of these programs? Second, a deeper understanding of the host immune response, and the role of sequential infection with different serovars, is urgently needed in order to inform vaccine development. Third, a rapid diagnostic test that can direct specific antibiotic therapy at the first encounter with the infected person would be a huge advance; no available test is currently sensitive and specific enough to recommend it in this context.

\section{Abbreviations}

CDC, Centers for Disease Control and Prevention; CI, confidence interval; EPM, expedited partner management; EPT, expedited partner therapy; hpi, hours post infection; LGV, lymphogranuloma venereum; MSM, men who have sex with men; NAAT, nucleic acid amplification test; PID, pelvic inflammatory disease; STI, sexually transmitted infection; USPSTF, US Preventive Screening Task Force.

\section{Disclosure}

The authors declare that they have no disclosures.

\section{Acknowledgments}

We thank Dana Kubilus for her assistance with preparing Figure 2.

\section{References}

I. Centers for Disease Control and Prevention (CDC): Sexually Transmitted Disease Surveillance, 20I2; 20I2. [http://www. cdc.gov/std/stats I 2/surv20 I 2.pdf]

2. Owusu-Edusei K, Jr., Chesson HW, Gift TL, Tao G, Mahajan R, Ocfemia MC, Kent CK: The estimated direct medical cost of selected sexually transmitted infections in the United States, 2008. Sex Transm Dis 2013, 40:197-201.

FlOOOPrime

RECOMMENDED

3. Gottlieb SL, Xu F, Brunham RC: Screening and treating Chlamydia trachomatis genital infection to prevent pelvic inflammatory disease: interpretation of findings from randomized controlled trials. Sex Transm Dis 2013, 40:97-102.

4. Gottlieb SL, Berman SM, Low N: Screening and treatment to prevent sequelae in women with Chlamydia trachomatis genital infection: how much do we know? J infect Dis 2010, 201 (Suppl 2):SI56-67.

5. Centers for Disease Control and Prevention: Sexually transmitted disease treatment guidelines. MMWR Morb Mortal Wkly Rep 2010, 59.

FlOOOPrime

6. Meyers DS, Halvorson H, Luckhaupt S: Screening for chlamydial infection: an evidence update for the U.S. Preventive Services Task Force. Ann Intern Med 2007, I47:I35-42.

$$
\text { FlOOOPrime }
$$$$
\text { RECOMMENDED }
$$

7. Centers for Disease Control and Prevention (CDC): Chlamydia screening among sexually active young female enrollees of health plans-United States, 2000-2007. MMWR Morb Mortal Wkly Rep 2009, 58:362-5.

8. Miller WC, Zenilman JM: Epidemiology of chlamydial infection, gonorrhea, and trichomoniasis in the United States-2005. Infect Dis Clin North Am 2005, 19:281-96.

\section{FlOOOPrime
RECOMMENDED}

9. Brunham RC: Immunity to Chlamydia trachomatis. J Infect Dis 20I3, 207:1796-7.

FlOOOPrime

RECOMMENDED 
10. Brunham RC, Pourbohloul B, Mak S, White R, Rekart ML: The unexpected impact of a Chlamydia trachomatis infection control program on susceptibility to reinfection. J Infect Dis 2005, 192:1836-44.

\section{FlOOOPrime}

1I. Sexual and reproductive health: Global incidence and prevalence of selected curable sexually transmitted infection, 2008. World Health Organization (WHO); 20I2. [http://www.who.int/ reproductivehealth/publications/rtis/stisestimates/en/]

12. Parish WL, Laumann EO, Cohen MS, Pan S, Zheng H, Hoffman I, Wang $\mathrm{T}, \mathrm{Ng} \mathrm{KH}$ : Population-based study of chlamydial infection in China: a hidden epidemic. JAMA 2003, 289: $1265-73$.

13. Unemo M, Clarke IN: The Swedish new variant of Chlamydia trachomatis. Curr Opin Infect Dis 20II, 24:62-9.

\section{FlOOOPrime}

14. Koper NE, van der Sande MA, Gotz HM, Koedijk FD, Dutch STIC: Lymphogranuloma venereum among men who have sex with men in the Netherlands: regional differences in testing rates lead to underestimation of the incidence, 2006-2012. Euro Surveill 2013, 18.

\section{FlOOOPrime}

\section{RECOMMENDED}

15. Ward H, Alexander S, Carder C, Dean G, French P, Ivens D, Ling C, Paul J, Tong W, White J, Ison CA: The prevalence of lymphogranuloma venereum infection in men who have sex with men: results of a multicentre case finding study. Sex Transm Infect 2009, 85:173-5.

\section{FIOOOPrime}

16. de Vrieze $\mathrm{NH}$, van Rooijen M, Schim van der Loeff MF, de Vries HJ: Anorectal and inguinal lymphogranuloma venereum among men who have sex with men in Amsterdam, The Netherlands: trends over time, symptomatology and concurrent infections. Sex Transm Infect 2013, 89:548-52.

\section{FloOOPrime \\ RECOMMENDED}

17. Patton ME, Kidd S, Llata E, Stenger M, Braxton J, Asbel L, Bernstein K, Gratzer B, Jespersen M, Kerani R, Mettenbrink C, Mohamed M, Pathela P, Schumacher C, Stirland A, Stover J, Tabidze I, Kirkcaldy RD, Weinstock H: Extragenital gonorrhea and chlamydia testing and infection among men who have sex with men-STD Surveillance Network, United States, 2010-20 I2. Clin Infect Dis 20I4, 58:1564-70.

\section{FlOOOPrime}

18. Singh D, Fine DN, Marrazzo JM: Chlamydia trachomatis infection among women reporting sexual activity with women screened in Family Planning Clinics in the Pacific Northwest, 1997 to 2005. Am J Public Health 20I I, I0 I: 1284-90.

19. Dunne EF, Chapin JB, Rietmeijer CA, Kent CK, Ellen JM, Gaydos CA, Willard NJ, Kohn R, Lloyd L, Thomas S, Birkjukow N, Chung S, Klausner J, Schillinger JA, Markowitz LE: Rate and predictors of repeat Chlamydia trachomatis infection among men. Sex Transm Dis 2008, 35:S40-4.

\section{FlOOOPrime}

\section{RECOMMENDED}

20. Gaydos CA, Wright C, Wood BJ, Waterfield G, Hobson S, Quinn TC: Chlamydia trachomatis reinfection rates among female adolescents seeking rescreening in school-based health centers. Sex Transm Dis 2008, 35:233-7.

\section{FlOOOPrime}

21. Hoover KW, Tao G, Nye MB, Body BA: Suboptimal adherence to repeat testing recommendations for men and women with positive Chlamydia tests in the United States, 2008-20 I0. Clin Infect Dis 2013, 56:51-7.

\section{FlOOOPrime}

22. Morrison RP, Caldwell HD: Immunity to murine chlamydial genital infection. Infect Immun 2002, 70:274I-5I.

23. Morrison SG, Morrison RP: The protective effect of antibody in immunity to murine chlamydial genital tract reinfection is independent of immunoglobulin A. Infect Immun 2005, 73:6I83-6.

24. Geisler WM, Lensing SY, Press CG, Hook EW, $3^{\text {rd }}$ : Spontaneous resolution of genital Chlamydia trachomatis infection in women and protection from reinfection. J Infect Dis 2013, 207: I850-6.

\section{FlOOOPrime}

25. Scholes D, Stergachis A, Heidrich FE, Andrilla H, Holmes KK, Stamm WE: Prevention of pelvic inflammatory disease by screening for cervical chlamydial infection. N EnglJ Med 1996, 334:1362-6.

26. Oakeshott P, Kerry S, Aghaizu A, et al.: Randomised controlled trial of screening for Chlamydia trachomatis to prevent pelvic inflammatory disease: the POPI (prevention of pelvic infection) trial. $B M J$ 2010, 340:cl642.

\section{FlOOOPrime
RECOMMENDED}

27. By P, Papp JR, Schachter J, Gaydos CA, Van Der Pol B; Centers for Disease Control: Recommendations for the Laboratory-Based Detection of Chlamydia trachomatis and Neisseria gonorrhoeae - 2014. MMWR Recomm Rep 2014, 63:I-19.

28. Schwebke JR, Rompalo A, Taylor S, Seña AC, Martin DH, Lopez LM, Lensing S, Lee JY: Re-evaluating the treatment of nongonococcal urethritis: emphasizing emerging pathogens-a randomized clinical trial. Clin Infect Dis 2011, 52:163-70.

\section{FlOOOPrime}

RECOMMENDED

29. Kong FY, Tabrizi SN, Law M, Vodstrcil LA, Chen M, Fairley CK, Guy R, Bradshaw C, Hocking JS: Azithromycin Versus Doxycycline for the Treatment of Genital Chlamydia Infection: A Meta-analysis of Randomized Controlled Trials. Clin Infect Dis 2014, 59:193-205.

\section{FlOOOPrime}

\section{RECOMMENDED}

30. Lau CY, Qureshi AK: Azithromycin versus doxycycline for genital chlamydial infections: a meta-analysis of randomized clinical trials. Sex Transm Dis 2002, 29:497-502.

3I. Handsfield HH: Questioning azithromycin for chlamydial infection. Sex Transm Dis 20I I, 38:1028-9.

32. Horner PJ: Azithromycin antimicrobial resistance and genital Chlamydia trachomatis infection: duration of therapy may be the key to improving efficacy. Sex Transm Dis 2012, 88:154-6.

33. Suchland RJ, Sandoz KM, Jeffrey BM, Stamm WE, Rockey DD: Horizontal transfer of tetracycline resistance among Chlamydia spp. in vitro. Antimicrob Agents Chemother 2009, 53:4604-I I.

34. Geisler WM, Pascual ML, Mathew J, Koltun WD, Morgan F, Batteiger BE, Mayes A, Tao S, Hurwitz SJ, Sayada C, Schinazi RF: Randomized, Double-Blind, Multicenter Safety and Efficacy Study of Rifalazil Compared with Azithromycin for Treatment of Uncomplicated Genital Chlamydia trachomatis Infection in Women. Antimicrob Agents Chemother 2014, 58:40 I4-9.

\section{FlOOOPrime}

\section{RECOMMENDED}

35. Golden MR, Whittington WL, Handsfield HH, Hughes JP, Stamm WE, Hogben M, Clark A, Malinski C, Helmers JR, Thomas KK, Holmes KK: Effect of expedited treatment of sex partners on recurrent or persistent gonorrhea or chlamydial infection. N Engl J Med 2005, 352:676-85 